\title{
Arctan Power Frequency Droop for Power Electronics Dominated Microgrids
}

\author{
C.N. Rowe ${ }^{\dagger 12}$, T.J. Summers ${ }^{\ddagger 1}$ and R.E. Betz ${ }^{* 1}$ \\ ${ }^{1}$ School of Electrical Engineering and Computer Science \\ University of Newcastle, Australia, 2308 \\ ${ }^{2}$ CSIRO Energy Centre \\ Steel River, Newcastle, NSW \\ email: ${ }^{\dagger}$ Christopher.Rowe@newcastle.edu.au; ${ }^{\dagger}$ Terry.Summers@ newcastle.edu.au; \\ *Robert.Betz@newcastle.edu.au.
}

\begin{abstract}
-
The microgrid is an environment constructed with the aim of controlling local distributed resources. The most common power flow control technique utilised inside a power electronics dominated or standalone microgrid is- a technique known as power frequency droop. This paper develops a novel droop controller based on an arctan power profile that can provide natural limiting of frequency deviations and accurate frequency output. The new control system has been simulated in SABER and results are presented that show the advantages of the control. A low voltage microgrid consisting of two inverters and a passive load has been constructed. Experimental results from the hardware system are presented to confirm the operation of the new droop controller.

Index Terms-

Distributed Generation, Microgrids, Power Electronics, Power Frequency Droop.
\end{abstract}

\section{INTRODUCTION}

The existing power system topology is arranged to minimise transmission losses whilst transporting power from a few central generators to the end loads. The distribution system also provides interconnections of radial feeders to provide an $\mathrm{N}-1$ contingency at all points on the system. The existing power system has been the subject of extensive research in areas such as power flow, protection systems and fault detection. However new methods of power production have become more prevalent, posing new questions of power engineers. Driven by predictions of peak oil production, dating back to 1956 [1], coupled with the contemporary proof of climate change [2], installation of distributed generation (DG) units are increasing. Renewable resources may provide a viable long term alternative to fossil fuel based generation. Along with sustainability there are further positives to DG units including the potential for cogeneration such as combined heat and power plants and the physical location of generators near loads, leading to decreased transmission losses.

Despite the numerous positive impacts of DG there are also many problems with its integration into existing power systems. A DG unit placed on a distribution feeder causes a higher fault current, requires changes to recloser settings and increases the frequency with which fuses blow [3]. The transient stability of various different DG units is examined in [4]. It is found that the impact of DG units on transient stability has mixed results, depending on the generator type and power electronics interface.

These problems highlight the need for an appropriate environment in which to develop and control DG technologies. Lasseter noted the "Application of individual distributed generators can cause as many problems as it may solve" before presenting the concept of a microgrid in [5]. By characterising the microgrid as a small autonomous unit within a power system, we create an environment where solutions can be formed on a microgrid level whilst mitigating the need for major augmentations to the distribution network. A microgrid may have the capacity to isolate itself during transients or faults and reconnect when the power supply is stable. Further, hierarchical control can be implemented to more clearly define the responsibilities of involved parties from distribution companies to microgrid operators [6]. The most widely accepted method to ensure the power sharing is maintained in microgrids involves drooping the frequency of supply relative to the power supplied by each DG unit.

This paper begins by introducing the existing theory on power - frequency and reactive power - voltage droop. After exploring the existing theory the new arctan algorithm is introduced and the new control scheme discussed. A discussion is provided on the benefits of this approach. The system is described and results are presented showing the operation of the control algorithm. 


\section{The EXIsting THEORY}

This paper is based on the implementation of the traditional power frequency droop method discussed in [6], [7] and [8]. This general approach is derived from the power flow across a single impedance. Two assumptions are made to simplify the power flow equations. It is assumed the $\mathrm{X} / \mathrm{R}$ ratio is large and that the power angle $(\delta)$ is small enough to apply the small angle criteria, $\sin (\delta) \approx \delta$ and $\cos (\delta) \approx 1$. Under these assumptions it can be shown that the power angle $(\delta)$ is proportional to the real power flow, as given in (1). Further it can be shown the voltage across the impedance $\left(U_{1}-U_{2}\right)$ is proportional to the reactive power flow, as given in (2). Where $U_{1}$ and $U_{2}$ are the voltages on either side of the impedance through which the power is flowing. A succinct proof of this theory is provided in [8].

$$
\begin{gathered}
\delta \approx \frac{X P}{U_{1} U_{2}} \\
U_{1}-U_{2} \approx \frac{X Q}{U_{1}}
\end{gathered}
$$

The general accepted form of the P-F and $\mathrm{Q}-\mathrm{V}$ droop equations is given in (3) and (4). The frequency is decreased relative to the real power supplied and the voltage increased relative to the reactive power flow to the load.

$$
\begin{gathered}
f-f_{0}=-m_{P}\left(P-P_{0}\right) \\
U-U_{0}=-m_{Q}\left(Q-Q_{0}\right)
\end{gathered}
$$

The traditional control scheme in [7] performs an abc to $\alpha \beta$ transform on the three phase currents and voltages. Once the $\alpha \beta$ stationary frame quantities are obtained, the three phase instantaneous power can be calculated as provided in (5) and (6).

$$
\begin{aligned}
& P=v_{\alpha} i_{\alpha}+v_{\beta} i_{\beta} \\
& Q=v_{\beta} i_{\alpha}-v_{\alpha} i_{\beta}
\end{aligned}
$$

The regulation of the Point of Common Coupling (PCC) voltage is achieved by implementing a PID loop that also takes into account the $\mathrm{Q}-\mathrm{V}$ droop requirements of the control scheme. The voltage error that feeds the PID controller is provided in (7). This voltage PID loop controls the reactive power flow by altering the voltage output of the inverter relative to the PCC voltage. In (7); $V_{o}$ is the voltage set point, $\mathrm{V}$ is the measured PCC voltage, $\mathrm{Q}$ is the reactive power output and $Q_{o}$ is the reactive power set point.

$$
V_{e r r}=V_{o}-V-m_{Q}\left(Q-Q_{o}\right)
$$

The control of real power sharing is achieved by altering the relative droop gradients $\left(m_{p}\right)$ and the power set point $\left(P_{o}\right)$ for each inverter. Equation (3) describes the relationship between power calculated and operating frequency.

It is often necessary to limit this fixed gradient, for example when operating near the limits of allowable frequency, as excursions in power may cause a rapid change in frequency and cause the DG unit to operate outside the allowed frequency requirements. This change in gradient, $m_{p}$ and/or $m_{q}$, would be exacted by an overall system controller that dynamically adjusted the DG units set points within the microgrid [6].

\section{ARCTAN BASED CONTROL}

The new control scheme proposed in this paper removes the constant frequency droop slope and replaces it with an arctan based algorithm. By implementing this arctan based power profile the microgrid operator can ensure that the operation frequency of the microgrid is always within preset bounds. Dynamic droop adjustments are the subject of [9] and [10] with the aim to gain better control whilst implementing frequency and voltage bounding. These systems basically limit the gradient near the frequency bounds whilst utilising a fixed gradient. However, in a power electronic dominated microgrid it is possible to augment the droop profile, as long as the control throughout the microgrid is homogeneous. Currently the profile for droop is inherently limited to have a fixed concavity of zero. By allowing variance in both gradient and concavity of the power profile we can achieve natural frequency bounding independently from the overall system controller.

To provide unique solutions throughout the entire power profile, any monotonically increasing function was considered for the profile. A cubic function was first considered for this purpose but was abandoned, due to the computational requirements for the cubic solution and the difficulty in creating a horizontal asymptote.

The arctan function provides adequate control over the gradient of droop about the power set point, has desirable horizontal asymptotes and existing function libraries in most coding languages. The new frequency droop equation was characterised as shown in (8). Where $f$ is the operating frequency of the inverter, $f_{o}$ is the frequency set point, $\rho$ is the arctan droop coefficient, $\mathrm{P}$ is the output power of the inverter and $P_{o}$ is the power set point.

$$
f=f_{o}-\frac{1}{\pi}\left(\arctan \left(\rho\left(P-P_{o}\right)\right)\right)
$$

By characterising the function in this way it is naturally bounded in the frequency domain from $\left(f_{o}+0.5\right) \mathrm{Hz}$ to $\left(f_{o}-0.5\right) \mathrm{Hz}$. The control over the gradient (and inherently concavity) is exacted by changing $\rho$. It is worthy of note that 


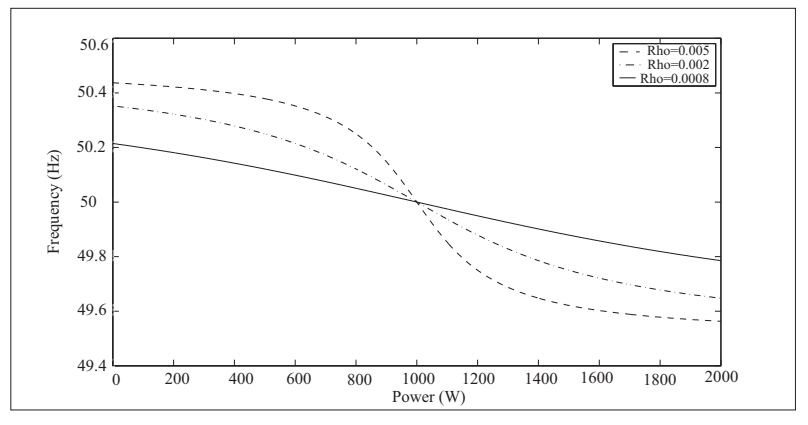

Figure 1. The effect of variations in $\rho$

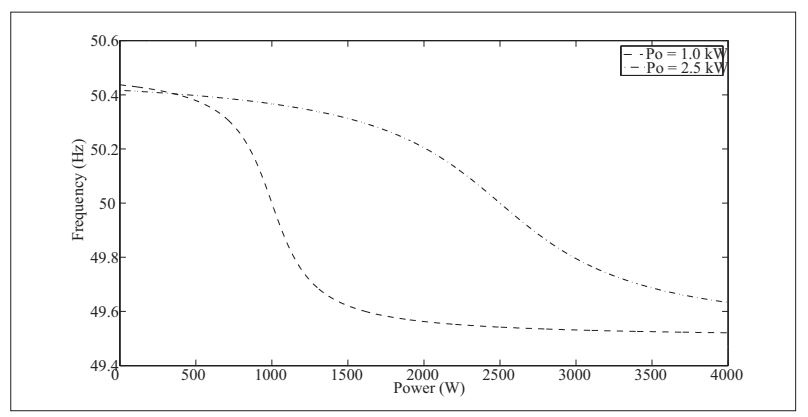

Figure 2. Two DGs with different $P_{o}$ set points

under the application of the small angle criteria, the arctan algorithm reduces to the same direct $\delta \propto P$ relationship as the general form of droop given in (1). The effect of variations in $\rho$ are plotted in Fig. 1. Also Fig. 2 shows a typical two inverter power sharing profile.

In [11], Li notes that the traditional way to improve power sharing performance between parallel inverters is to increase the gradient of droop. Essentially the arctan function has a larger tangential gradient than traditional drop around the power set point thus achieving a faster response. The corollary is of course also true. That at the frequency bounds the arctan droop will have a slower response. As higher level controllers are generally employed to dynamically adjust the $P_{o}$ operating point, the slower response at the frequency bounds is not a major point of contention and may be a desirable property of the control scheme.

The new control scheme for arctan droop is depicted in Fig. 3. The control scheme discussed in Section II is augmented to include the new arctan based frequency droop. A Clark transformation is performed on the three phase currents and voltages to obtain two phase $\alpha \beta$ quantities. The real and reactive power are calculated from the $\alpha \beta$ currents and voltages. Based on the power flow, the voltage magnitude is controller by a PID loop as provided in (7). Similarly the frequency of the output

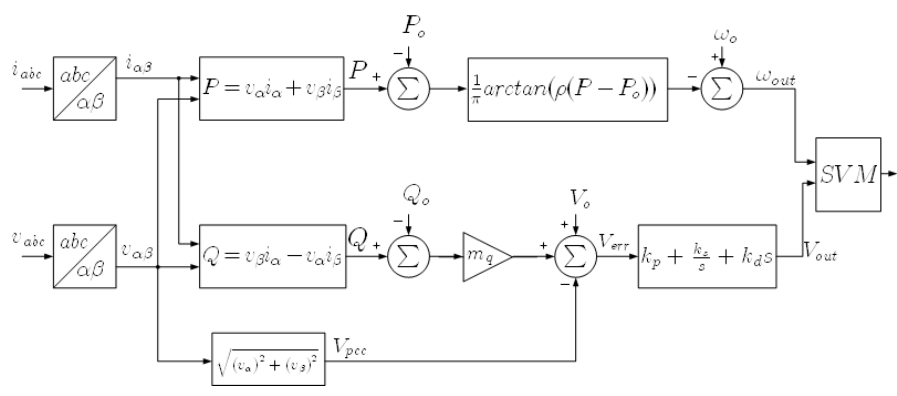

Figure 3. Control Block Diagram

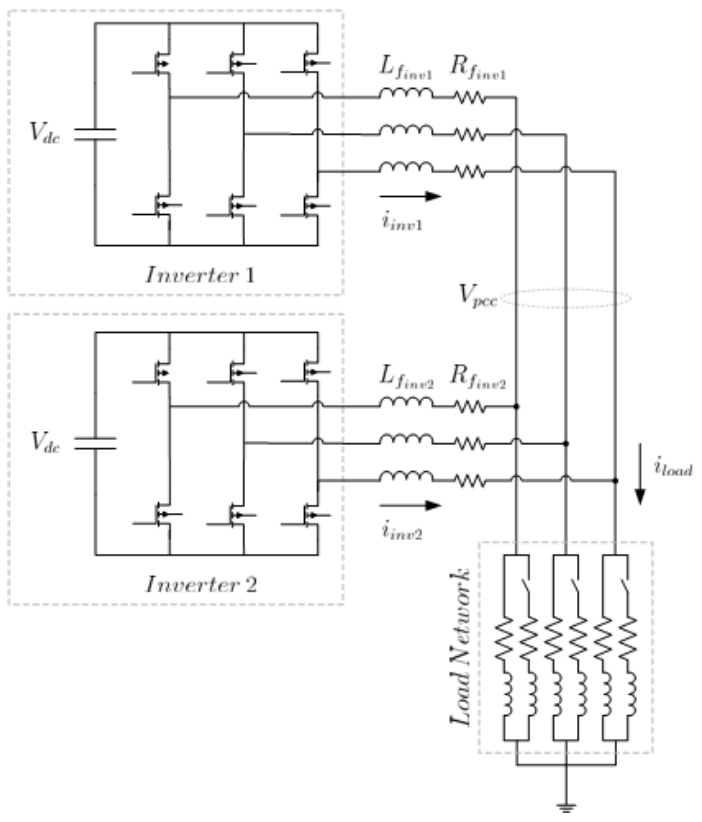

Figure 4. System Configuration

is determined by the new arctan algorithm of (8).

\section{EXPERIMENTAL SYSTEM}

The new control scheme was implemented in a two-inverter microgrid. The microgrid system is shown in Fig. 4. A hardware system was constructed and a simulation model of the hardware system.

\section{A. SABER ${ }^{\circledR}$ Simulation}

The simulation was performed in the SABER $^{\circledR}$ simulation package. The modeling uses ideal logic driven switches within the inverter. The bus voltage was simply derived from a voltage source.

The new control algorithm was implemented in a dynamic link library written in the ' $C$ ' language. In this way the control algorithm emulates a Digital Signal Processor (DSP) based 
Table I

SYSTEM PARAMETERS

\begin{tabular}{|c|c|}
\hline Parameter & Nominal Value \\
\hline \hline$V_{l-l}$ & $415 \mathrm{~V}$ \\
\hline$P_{\text {rated Inverter } 1}$ & $2 \mathrm{kVA}$ \\
\hline$P_{\text {rated Inverter } 2}$ & $36 \mathrm{kVA}$ \\
\hline$L_{\text {f Inverter } 1}$ & $53.0 \mathrm{mH}$ \\
\hline$R_{\text {f Inverter } 1}$ & $0.5 \Omega$ \\
\hline$L_{\text {f Inverter } 2}$ & $53.0 \mathrm{mH}$ \\
\hline$R_{\text {f Inverter } 2}$ & $0.5 \Omega$ \\
\hline$P_{\text {Load Network }}$ & $0 \longleftrightarrow 4 \mathrm{~kW}$ \\
\hline$Q_{\text {Load Network }}$ & $0 \longleftrightarrow 2 \mathrm{kVAr}$ \\
\hline
\end{tabular}

system. The dynamic link library is executed once every $250 \mu s$, emulating a DSP running with a main control loop speed of $4.0 \mathrm{kHz}$. Additionally Space Vector Modulation (SVM) was utilised to map from the alpha-beta frame directly to firing times [12]. Pulse centred zero SVM was implemented as the modulation scheme.

The model of the passive load contains only resistive and inductive elements. The implementation of load variations in this way models the possible load variations achievable in the hardware, on which the control scheme will be applied.

\section{B. Hardware}

The hardware system consists of two inverters in parallel, connected to a passive resistive and inductive load. The first inverter is a CS-IIB four leg inverter from Creative Power Technologies. It utilises MOSFET switches and the controller is a fixed-point Texas Instruments (TI) Digital Signal Processor (DSP). The fourth leg of the inverter is currently not connected. The second device is a three leg inverter designed and built by the Electrical Engineering department at The University of Newcastle. It utilises IGBT switches and a floating-point TI DSP.

The parameters of the system and ratings of each device are provided in Table I which also provides the various ratings of devices. The filter inductors in the hardware system are $53 \mathrm{mH}$ to mimimise transient reactive power flow during synchronisation. In the simulation the filter inductors are decreased to 16 $\mathrm{mH}$ without any change in the effectiveness of the control. It is expected that the hardware filter inductance can be decreased in the future with better synchronisation algorithms. The load is composed of passive resistive and inductive elements that are switched to simulate step transients in power demand.

\section{RESULTS}

The SABER ${ }^{\circledR}$ simulation configuration has been described in the above section. This system was used to simulate the response of the controller to step changes in power demand. Fig. 5 shows

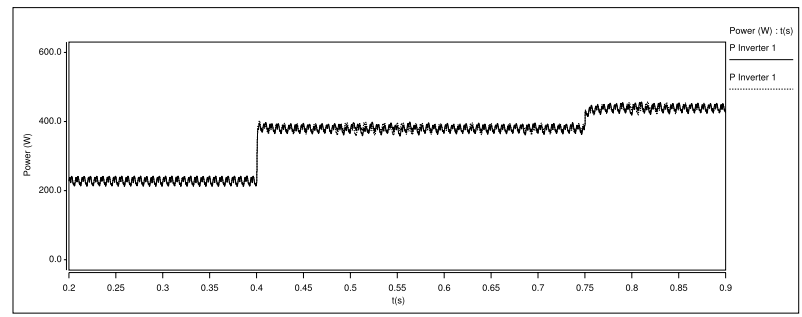

Figure 5. Three step changes in load with matched parameters

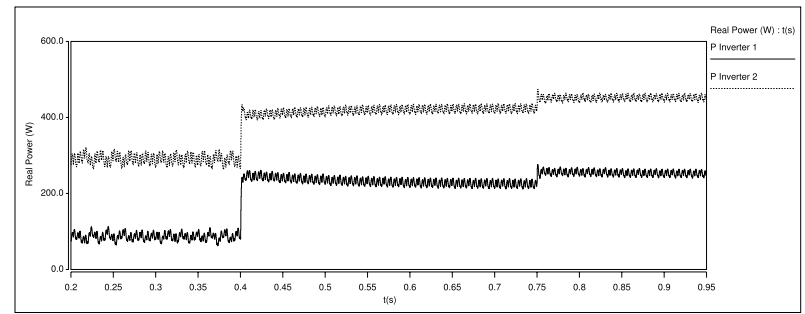

Figure 6. Fixed gradient droop - Three step response

the arctan droop with matched $\rho$ 's and a power set point of $0 \mathrm{~W}$ it is clear that the first transient is very fast while the second is slower. This is due to the steeper droop gradient about the set point of $0 \mathrm{~W}$.

A comparison is provided between normal fixed gradient droop and arctan droop in Fig. 6 and Fig. 7. In these plots the power set point of Inverter 1 and 2 were 220 and $420 \mathrm{~W} / \mathrm{ph}$ respectively. Note that the arctan droop settles to a steady state value around 0.55 seconds whilst the fixed gradient droop does not settle until after 0.6 seconds. It also provides a better transient in the second small step change in power. Fig. 8 also shows the system voltages and inverter two currents during the last small step change in demand.

A simulation was performed to observe the interaction between arctan and fixed gradient control. Inverter 1 was programmed with the arctan gradient whilst inverter 2 was programmed with the fixed gradient droop. Again the power set point of Inverter 1 and 2 were 220 and $420 \mathrm{~W} / \mathrm{ph}$ respectively.

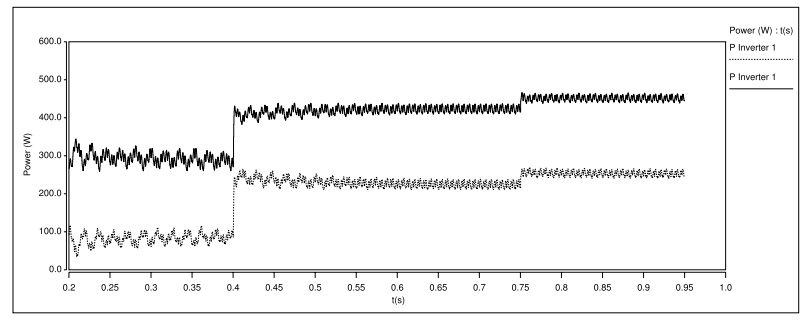

Figure 7. Arctan gradient - Three step response 


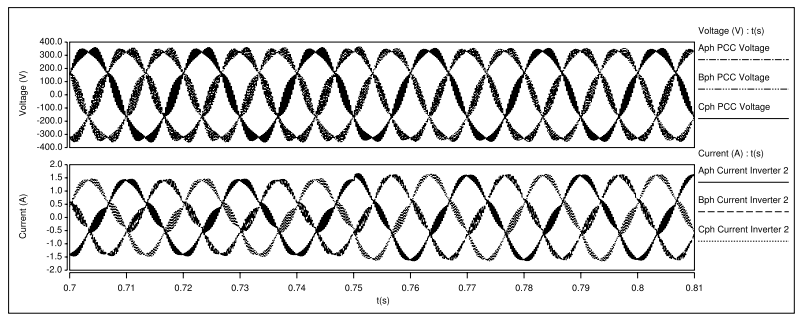

Figure 8. Three phase PCC voltages and Inverter 2 supply currents under small load change

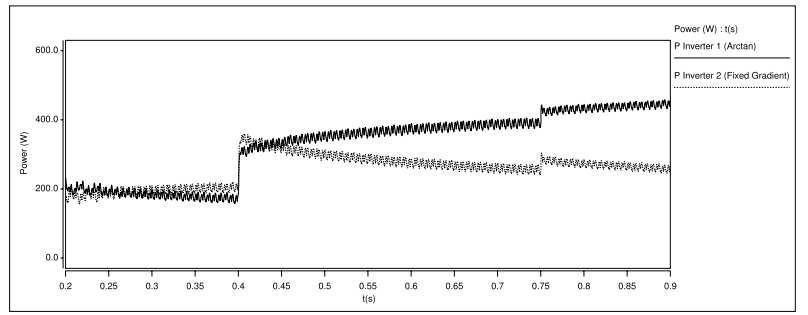

Figure 9. Arctan and fixed gradient droop interaction

It can be seen that the fixed gradient droop has a stronger control of the power flow at the start up driving itself towards its set point. After the first load change the arctan gradient is increased, as it is closer to its power set point, thus it has a stronger control over the power flow and drives itself to its power set point.

Fig. 10 confirms the correct operation of the droop, with the control frequency decreasing slightly with each increase in load. Also the arctan droop changes gradient such that at the bounds of frequency, the control system has less ability to control power flow. Fig. 11 shows this phenomenon, it can be seen in this graph that under a large step change in load demand the two inverters are both limited by the arctan profile. The power of inverter 1 and 2 become the same, despite their different set points as they are limited by the decrease in the gradient of the droop.

The hardware system has been previously described in Section IV-B. Preliminary results were recorded on a $500 \mathrm{MHz}$ oscilloscope at a nominal operating voltage of $20 \mathrm{~V}$ RMS and

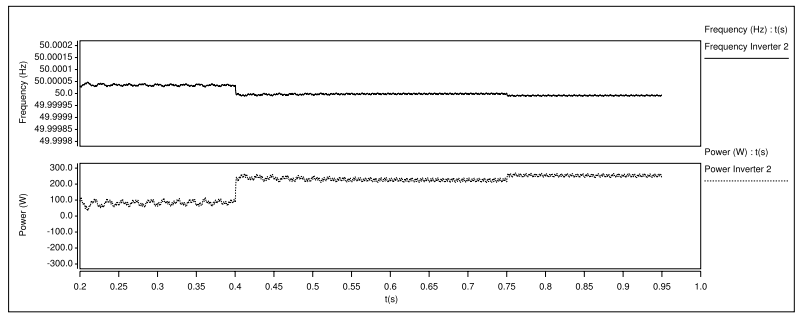

Figure 10. Frequency droop demonstration

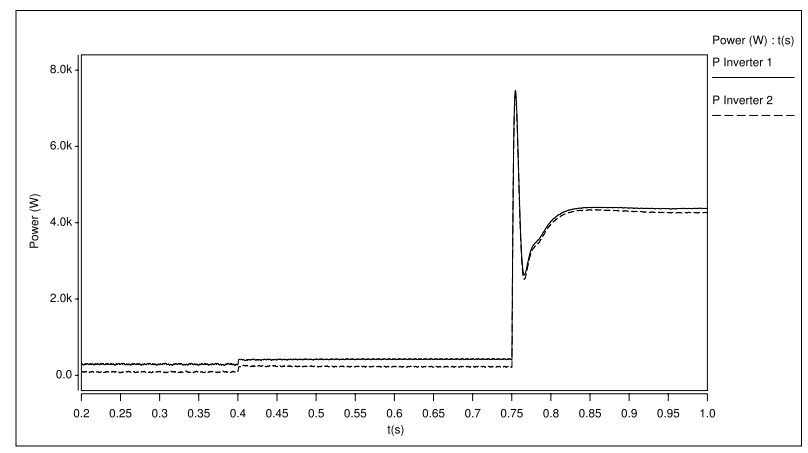

Figure 11. Limiting of power output under large power demands

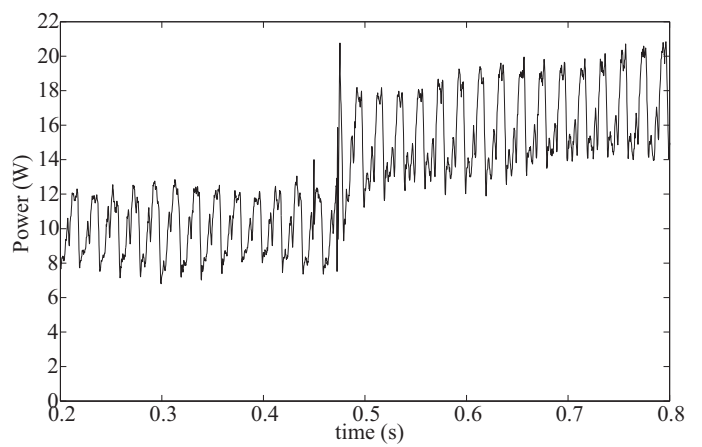

Figure 12. Inverter 1 Power Output

currents in the range of zero to two Amps RMS. A step change in load from $9 \mathrm{~W}$ to $18 \mathrm{~W}$ was applied to the two inverter system. Fig. 14 and Fig. 15 show the inverter 1 (channel2) and inverter 2 (channel3) 'B' phase currents before and after the step change in load. In these two plots the external current probes were set to $0.5 \mathrm{~A}$ /division. Channel 1 shown in Fig. 14 shows the PCC voltage, operating at $20 \mathrm{~V}$ RMS as required. It can be seen that the arctan algorithm correctly shares the voltage evenly between the phases.

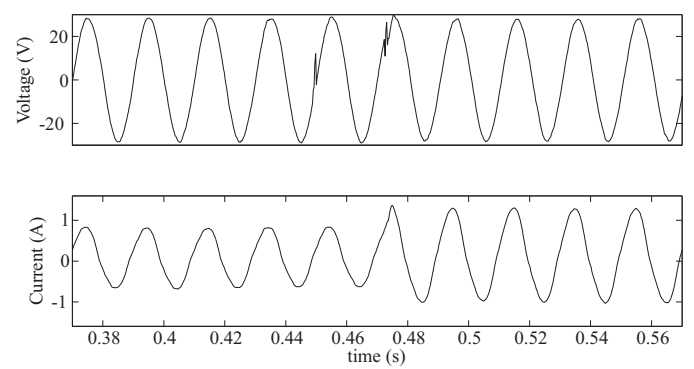

Figure 13. Current and voltage during power transient 


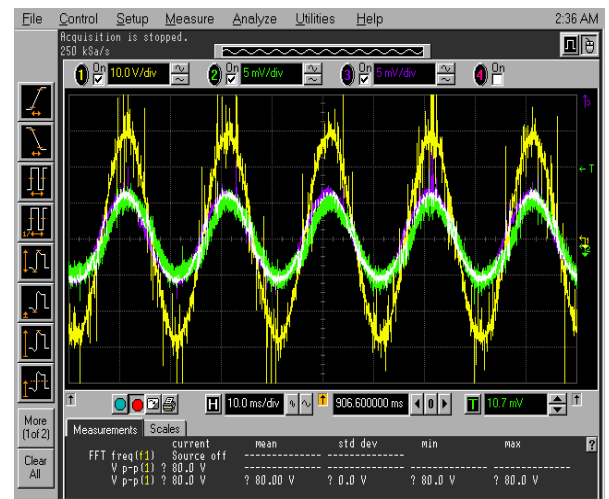

Figure 14. Inverter currents and PCC voltage before load change

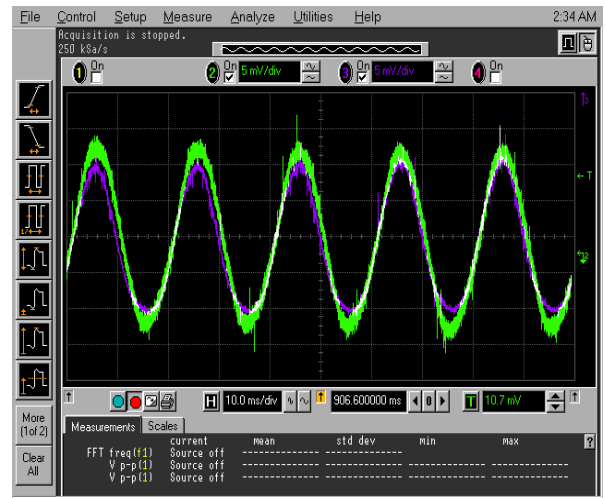

Figure 15. Inverter currents after load change

Fig. 12 and Fig. 13 show the power increase of inverter 1, the A phase voltage and A phase current under the same step change in load. These results were logged internally by inverter 1 . The increase in power correlates to that shown by the oscilloscope graphs and confirm the correct operation of the arctan droop.

\section{CONCLUSiOnS}

This paper extends on current microgrid control theory to contribute a novel arctan based droop control method. The new control system was simulated in the SABER ${ }^{\circledR}$ simulation package with results confirming the arctan profile provides a more accurate frequency response about the power set point and natural frequency limiting under large power demands. Hardware results were obtained from a three phase, two inverter microgrid and presented to show the correct operation of the arctan power frequency droop.

\section{REFERENCES}

[1] Hubbert, M. King, "Nuclear Energy and the Fossil Fuels," Drilling and Production Practice, 1956
[2] Weart, S.R, The Discovery of Global Warming. Cambridge, MA: Harvard University Press, 2003; revised and expanded edition, 2008, ISBN-13: 978067403189-0.

[3] McDermott, T.E.; Dugan, R.C.; , "PQ, reliability and DG," Industry Applications Magazine, IEEE , vol.9, no.5, pp. 17- 23, Sept.-Oct. 2003

[4] Reza, M.; Schavemaker, P.H.; Slootweg, J.G.; Kling, W.L.; van der Sluis, L.; , "Impacts of distributed generation penetration levels on power systems transient stability," Power Engineering Society General Meeting, 2004 IEEE , vol., no., pp.2150-2155 Vol.2, 10-10 June 2004

[5] Lasseter, R.H.; Paigi, P.; , "Microgrid: a conceptual solution," Power Electronics Specialists Conference, 2004. PESC 04. 2004 IEEE 35th Annual , vol.6, no., pp. 4285- 4290 Vol.6, 20-25 June 2004

[6] Guerrero, J.M.; Vasquez, J.C.; Teodorescu, R.; , "Hierarchical control of droop-controlled DC and AC microgrids - a general approach towards standardization," Industrial Electronics, 2009. IECON '09. 35th Annual Conference of IEEE , vol., no., pp.4305-4310, 3-5 Nov. 2009

[7] Chandorkar, M.C.; Divan, D.M.; Adapa, R.; , "Control of parallel connected inverters in standalone AC supply systems," Industry Applications, IEEE Transactions on , vol.29, no.1, pp.136-143, Jan/Feb 1993

[8] De Brabandere, K..; Bolsens, B..; Van den Keybus, J..; Woyte, A..; Driesen, J..; Belmans, R..; , "A Voltage and Frequency Droop Control Method for Parallel Inverters," Power Electronics, IEEE Transactions on , vol.22, no.4, pp.1107-1115, July 2007

[9] Hsin-Hsin Ho; Ke-Horng Chen; Wen-Tsao Chen; , "Dynamic Droop Scaling for Improving Current Sharing Performance in a System with Multiple Supplies," Circuits and Systems, 2007. ISCAS 2007. IEEE International Symposium on , vol., no., pp.545-548, 27-30 May 2007

[10] Aghasafari, M.A.; Lopes, L.A.C.; Williamson, S.; , "Frequency regulation and enhanced power sharing in microgrids including modified droop coefficients and virtual resistances," Electrical Power \& Energy Conference (EPEC), 2009 IEEE , vol., no., pp.1-6, 22-23 Oct. 2009

[11] Yun Wei Li; Ching-Nan Kao;, ,An Accurate Power Control Strategy for Power-Electronics-Interfaced Distributed Generation Units Operating in a Low-Voltage Multibus Microgrid," Power Electronics, IEEE Transactions on , vol.24, no.12, pp.2977-2988, Dec. 2009

[12] Betz, R.E.; Cook, B.J.; Henriksen, S.J.; , "A digital current controller for three phase voltage source inverters," Industry Applications Conference, 1997. Thirty-Second IAS Annual Meeting, IAS '97., Conference Record of the 1997 IEEE , vol.1, no., pp.722-729 vol.1, 5-9 Oct 1997 